\title{
Gestión del Conocimiento en Cadenas Productivas. El Caso de la Cadena Láctea en Colombia
}

\author{
Mónica M. Simanca ${ }^{(1)}$, Luz A. Montoya ${ }^{(2)}$ y Cesar A. Bernal ${ }^{(3)}$ \\ (1) Fac. de Ingeniería, Dpto Ingeniería de Alimentos, Universidad de Córdoba, Carrera 6 No. 76-103, \\ Montería - Colombia. (e-mail: msimanca@correo.unicordoba.edu.co) \\ (2) Fac. de Minas, Escuela Ingeniería de la Organización, Universidad Nacional de Colombia, Edificio M8B \\ 99-07, Sede Medellín. Colombia (e-mail: lamontoyar@unal.edu.co) \\ (3) Escuela Internacional de Ciencias Económicas y Administrativas, Universidad de La Sabana. Bogotá - \\ Colombia. (e-mail: cesar.bernal@unisabana.edu.co)
}

Recibido Oct. 2, 2015; Aceptado Nov. 25, 2015; Versión final Feb. 19, 2016, Publicado Jun. 2016

\begin{abstract}
Resumen
Este artículo presenta un modelo para el análisis de la gestión del conocimiento en la cadena productiva de lácteos en Colombia. Se evaluaron elementos facilitadores, el proceso de creación y almacenamiento del conocimiento, y la competitividad en 349 empresas. Se utilizó un instrumento de medición con una escala Likert con puntuaciones de 1 a 5 . El modelo muestra que el concepto de cadena productiva es algo más que un eslabonamiento entre los actores del sector productivo, el estado y la academia. Para una adecuada gestión del conocimiento es necesario tener claridad sobre los facilitadores y la funcionalidad del proceso de gestión del conocimiento como estrategia para mejorar la competitividad de la cadena. El modelo contribuye a generar análisis más profundos en el área de gestión del conocimiento. También apoya la toma de decisiones en la cadena láctea colombiana y puede servir de referente para otros sectores con similares condiciones.
\end{abstract}

Palabras clave: gestión del conocimiento; modelo; cadena productiva; cadena láctea colombiana; competitividad

\section{Knowledge Management in Production Chains. The Case of the Dairy Chain in Colombia}

\begin{abstract}
This paper presents a model for analyzing knowledge management in the dairy productive chain in Colombia. Enabling elements, the process of creating and storing knowledge and competitiveness in 349 companies, were evaluated. This was done by using a measuring instrument with a Likert scale with scores from 1 to 5 . The model shows that the concept of productive chain is more than a linking between the actors of the productive sector, the state and academia. To ensure effective management of knowledge it is necessary to be clear about the enabling elements and the functionality of the process of knowledge management as a strategy to enhance the competitiveness of the chain. The model contributes to generate deeper analysis in the area of knowledge management. It also supports decision making in the colombian dairy chain and can serve as a model for other sectors with similar conditions.
\end{abstract}

Keywords: knowledge management; model; productive chain; colombian dairy chain; competitiveness 


\section{INTRODUCCIÓN}

El conocimiento ha marcado el desarrollo de la humanidad, y en la actualidad se considera un factor importante que condiciona la competitividad en las empresas (Capó et al., 2007). Es por ello que ha sido considerado como factor de desarrollo e innovación (Andreou et al., 2007; Cantner et al., 2009); y la presente generación es reconocida por la importancia que le confiera al conocimiento como elemento clave para el mejoramiento de sus resultados. La gestión del conocimiento se concibe como una importante herramienta que ejerce efectos positivos en la innovación y el rendimiento empresarial (López y Meroño, 2011); y para su gestión se utilizan modelos que en algunas ocasiones resulta difícil de aplicar y manipular (OECD, 2003). En los últimos dos decenios la literatura académica y empresarial que incorpora el término gestión del conocimiento se ha incrementado (Benavides y Quintana, 2005; Bueno, 1999; Soto y Barrios, 2006); Sin embargo, no hay un consenso que adopte una definición generalizada, debido a las perspectivas de concepción de la disciplina y el proceso de evolución de la actividad (Martínez, 2011).

No obstante, el no consenso sobre una definición generalizada de lo que es la gestión del conocimiento, si lo hay sobre los impactos positivos de esta gestión tanto para las personas, las organizaciones, las cadenas productivas, los clústeres, las regiones y las propias naciones (Drucker, 1994; Nonaka y Takeuchi,1995 y Barney, 2003). Por este motivo la importancia de analizar este tema en el ámbito de las cadenas productivas en contextos de países en desarrollo como Colombia, en donde estas cadenas comienzan a ser consideradas como estratégicas para la competitividad empresarial, regional y nacional (Reyes, 2005 y Porter, 2009).

Las cadenas productivas son concebidas como un modo de integración, a través de estructuras cooperativas que permiten el fortalecimiento económico y tecnológico, y promueven la consolidación de tejidos sociales (Simanca y Montoya, 2014); si bien estas cadenas han sido consideradas pertinentes para la organización del sector agropecuario colombiano, no son las únicas formas de integración empresarial, ya que se tienen los conglomerados (clusters), alianzas estratégicas, parques tecnológicos, parques industriales, redes empresariales (networks), chaebol, kibutz, entre otros, las cuales pueden coexistir y en conjunto aportar elementos particulares en cada uno de los sectores a los que pertenecen (Castellanos et al., 2009).

La cadena productiva de lácteos en Colombia consiste en un sistema complejo de seis eslabones, donde el producto básico es la producción de leche, proveniente del ganado bovino de sistemas especializados o de doble propósito. Los eslabones que interactúan y se interrelacionan en la cadena láctea colombiana son: los proveedores de bienes y servicios, las unidades productivas, los centros de acopio, los industriales, los intermediarios y el consumidor final (Mojica et al., 2007). El sector lácteo colombiano es reconocido por su alto potencial exportador de productos con valor agregado y se le atribuye una importancia estratégica, económica, social, de seguridad alimentaria para la población colombiana y motor de desarrollo local, regional y nacional (República de Colombia-Departamento Nacional de Planeación, 2010); razón por la cual es uno de los priorizados por la política pública colombiana (Consejo Nacional Lácteo, 2010). En Colombia no se dispone de una herramienta para el análisis de la gestión del conocimiento en la cadena productiva de lácteos, por lo que en la presente investigación se hace una propuesta metodológica con el fin de aportar herramientas de gestión y análisis que permitan mejorar su competitividad.

\section{CADENA PRODUCTIVA}

López (2003) define una cadena productiva como un sistema conformado por la interacción en armonía entre diversos participantes, directa o indirectamente, en la producción y consumo de productos y servicios. Mientras que Lima et al., (2001) mencionan que las cadenas productivas son conjuntos de actores sociales, como sistemas productivos agropecuarios y agroforestales, proveedores de servicios e insumos, industrias de procesamiento y transformación, distribución y comercialización, además de consumidores finales del producto y subproductos. La Ley 811 de las Organizaciones de Cadenas, concibe una cadena como el conjunto de actividades que se articulan técnica y económicamente desde el inicio de la elaboración de un producto agropecuario hasta su comercialización final (Congreso de la Repúplica de Colombia, 2003). Estas organizaciones son coordinadas por el Ministerio de Agricultura y Desarrollo Rural (MADR), su inscripción está reglamentada mediante el Decreto 3800 de 2006 y la Resolución 186 de 2008, y su marco estratégico definido por los acuerdos de competitividad.

El proceso de globalización en el que se desenvuelve el mundo empresarial en la actualidad, tiene como una de sus principales consecuencias la importancia de las aglomeraciones y las características territoriales y económicas locales como factores claves para competir (Belussi y Sedita, 2009; Parrilli, 2009). En este sentido, son diversos los autores que desde diferentes enfoques sugieren que las aglomeraciones, especialmente las territoriales, benefician a las empresas, más aún, algunos enfatizan en la superioridad de 
esta forma de organización industrial en comparación con la de producción en masa y la empresa verticalmente integrada (Capó-Vicedo et al., 2011). Sin embargo, como señala Storpe (2005) el gran número de conceptos y aproximaciones a la caracterización de este tipo de aglomeraciones, produce confusión y dificulta la comprensión del tema. Así, diversos autores que han mostrado en las últimas décadas una creciente atención por las empresas localizadas en áreas geográficas específicas, proponen diversos enfoques para este propósito tales como: el enfoque de Distrito Industrial (Becattini, 2002), el de Cluster Industrial (Porter, 1991), el de Sistema Nacional de Innovación (Lundvall, 1992), el de Cadena de Suministro (Lambert et al., 1998, Frohlich y Westbrook, 2001; Calderón y Lario, 2007 y Salazar et al., 2012), el de Parques Tecnológico (Phan, Siegel y Wright, 2005), el de Redes o Networks (López, 2003) y el de Cadenas Productivas (Beckerman y Cataife, 2001 y Moncayo, 2002).

Para efectos de esta investigación se utilizó el enfoque de cadenas productivas, que es el enfoque que integra los conceptos clave de los otros relacionados con las aglomeraciones y el trabajo colaborativo interagentes o actores, en un proceso productivo, económico y social. Esto debido a que el mayor nivel de externalización y dinamismo del entorno, hace que las empresas ya no pueden competir como entidades individuales, sino que requieren competir como cadenas de valor (Frohlich y Westbrook, 2001). Por tanto, la creación de valor por parte de las empresas y de los sectores productivos tiene que fundamentarse en un enfoque integrador (Amit y Zott, 2001). Así, las empresas para competir requieren no sólo de la integración de sus procesos y la alineación de sus estrategias empresariales, sino también de la integración y la alineación de los procesos y estrategias entre empresas (Cagliano et al., 2006) y entre estas y las instituciones públicas, educativas (universidades, centros especializados de formación, entre otros actores), parques tecnológicos y servicios de información, reciclaje y apoyo técnico (Vila, Ferro y Rodríguez, 2000).

A este respecto, el enfoque de cadena productiva ofrece un marco conceptual para comprender la articulación de diferentes unidades empresariales y actores económicos y sociales en un contexto determinado, de cara al proceso de generación de valor y el papel que cumple cada una de las empresas y los actores económicos y sociales que intervienen en el mismo. Igualmente, la cadena productiva, como concepto, provee elementos importantes para el diseño de políticas de apoyo empresarial que favorecen la generación de riqueza a través de la consolidación de ventajas competitivas (Isaza, s.f.).

El concepto de cadena productiva no es equivalente al de cadena de valor, ni al de cadena de suministros; cadena productiva es un concepto que comenzó a utilizarse en Latinoamérica en la década de los noventas de siglo XXI, para referirse a trabajos colaborativos entre eslabones de producción y la articulación y el diseño de políticas sectoriales y de apoyo empresarial por parte de los organismos gubernamentales (Isaza, s.f. y Beckerman y Cataife, 2001). Así, desde los años 90's las cadenas productivas hacen parte del marco conceptual de competitividad que caracteriza a las políticas de desarrollo nacional y regional en América Latina (Moncayo, 2002).

Desde esta perspectiva, las cadenas productivas son una variante del enfoque de competitividad propuesto por Porter, que surge a partir de algunos trabajos realizados por Esser et al. (1996) para la Cepal (Isaza, s.f.). Este enfoque destaca que para la generación de ventajas competitivas no sólo se deben considerar los factores de tipo micro inherentes a la cadena de valor de la empresa, sino que surge de una interacción compleja y dinámica entre el nivel micro, el meso, que corresponde al conjunto de políticas de apoyo específico lideradas por el estado y los actores sociales; el macro, representado por el conjunto de políticas y condiciones macroeconómicas y, finalmente, el meta, el cual sintetiza el conjunto de comportamientos y normas que rigen lo jurídico, lo político y lo económico a través de la interacción e integración de diferentes actores sociales.

\section{Estructura de una Cadena Productiva}

En una cadena productiva existen elementos constitutivos, actores y actividades que definen su estructura y funcionamiento; entre los elementos que constituyen una cadena productiva están los eslabones, los segmentos, los flujos y los entornos organizacionales, los cuales son estructuras diferenciadas, interactuantes y que aportan a la construcción de un objetivo común. Los eslabones hacen referencia a actores involucrados en las actividades de la cadena; los segmentos son grupos de actores homogéneos dentro de un mismo eslabón; y los flujos permiten entender las relaciones entre los eslabones y segmentos, aumentando la comprensión de la cadena. Castellanos et al., (2009) sugiere la representación gráfica de los eslabones de las cadenas productivas colombianas por medio de rectángulos, los segmentos mediante rectángulos, círculos u otras formas y los flujos a través de flechas.

\section{Cadena Láctea}

La cadena productiva de lácteos en Colombia consiste en un sistema complejo de seis eslabones, donde el producto básico es la producción de leche, proveniente del ganado bovino de sistemas especializados o de 
doble propósito (Mojica et al., 2007). Los eslabones que interactúan y se interrelacionan en la cadena láctea colombiana son: los proveedores de bienes y servicios, las unidades productivas, los centros de acopio, los industriales, los intermediarios y el consumidor final; y entre los productos derivados se encuentran, mantequilla, queso, yogurt y preparados a base de leche como postres, dulces, entre otros (Espinal et al., 2005). En la figura 1 se ilustra la representación de la cadena láctea colombiana.

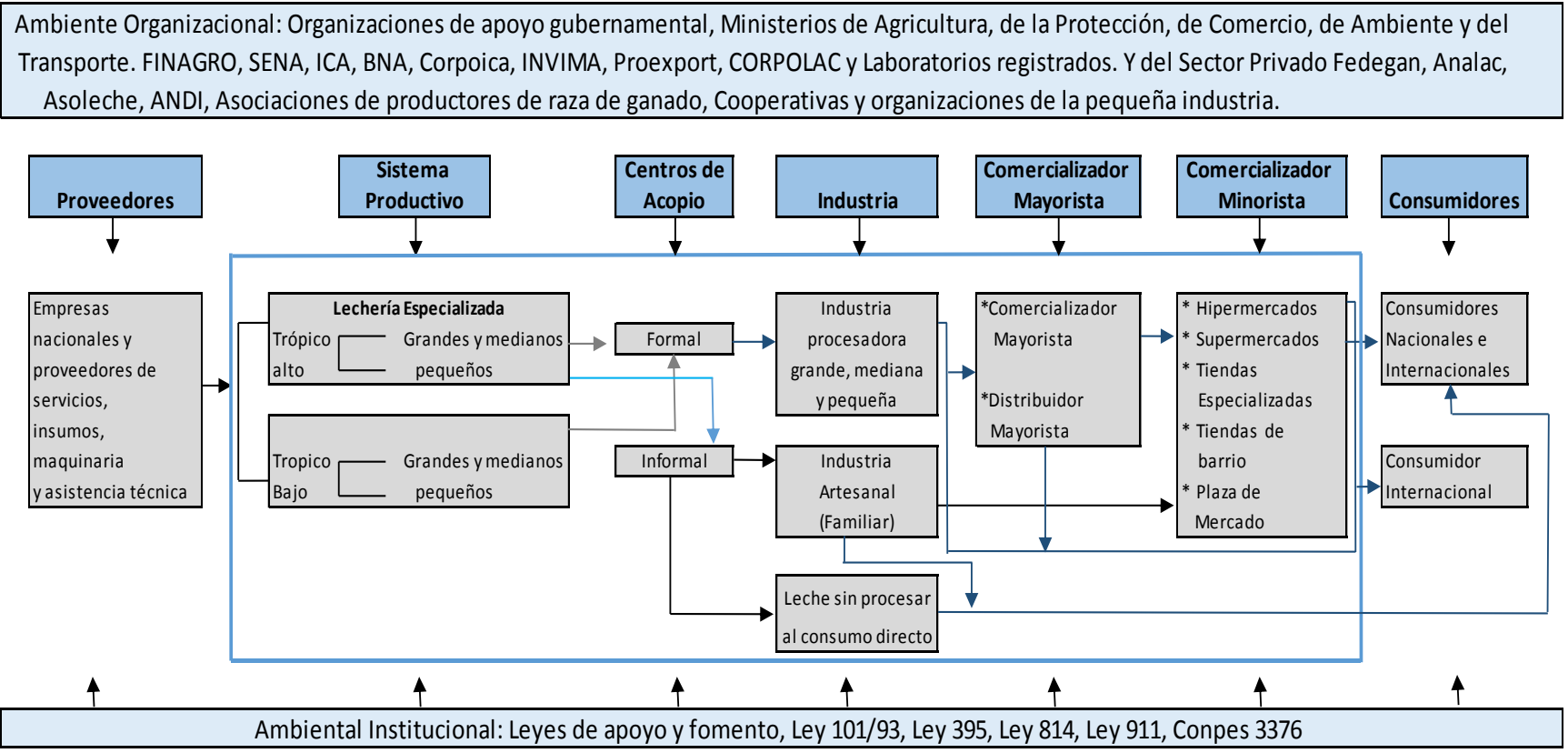

Fig. 1. Cadena láctea colombiana (Consejo Nacional Lácteo, 2010)

\section{GESTIÓN DEL CONOCIMIENTO}

El conocimiento es un concepto amplio y abstracto cuya definición ha sido objeto de debate desde tiempos remotos, en la actualidad se considera como una fuente de ventajas competitivas sostenible para la organización por lo que las prácticas de gestión de éstas se han centrado en su estudio (Alavi y Leidner, 2001). El conocimiento puede ser definido como una mezcla flexible de experiencias relacionadas, de valores, de información contextual y de visión experta que sirve como marco para la incorporación de nuevas experiencias e información, y es útil para la acción (Davenpork y Prusak, 1998). Para Nonaka y Takeuchi (1995), el conocimiento es un proceso humano dinámico que consiste en justificar las creencias personales en torno a la verdad.

En la literatura existen diversos autores que definen la gestión del conocimiento desde diferentes perspectivas; Briceño y Bernal (2010) señalan que aunque el término tiene un uso generalizado, el significado y enfoque del mismo se percibe de manera muy diversa en las organizaciones, principalmente en los países en vía de desarrollo, lo que dificulta su real comprensión y la evaluación de los procesos de implementación de la gestión del conocimiento. La gestión del conocimiento ha sido concebida desde tres perspectivas diferentes: una relacionada con la tecnología, que se encuentra fuertemente concentrada en el registro, codificación de datos y en la administración de documentos y que se identifica como la primera generación (Carrillo, 2001); una segunda perspectiva que lo relacionada con el talento humano; y por último la perspectiva que lo relaciona con las organizaciones (Del Moral et al., 2007).

Tarí y García (2013) consideran la gestión del conocimiento como un proceso dinámico de creación, almacenamiento y transferencia, y de aplicación y uso del conocimiento, con el fin de mejorar los resultados de una organización. Mientras que Fugate, et al., (2009) lo conciben como un proceso de generación, diseminación, compartimiento e interpretación de conocimiento. Ocampo y Castillo (2003), consideran que la gestión del conocimiento es una herramienta que aumenta la productividad mediante planteamientos y políticas específicas que llevan a un aprovechamiento máximo de los recursos; se refieren especialmente al aprovechamiento del conocimiento como capital intangible antes subutilizado.

\section{MODELOS DE GESTIÓN DEL CONOCIMIENTO}

La elaboración de modelos para la creación y gestión del conocimiento ha sido abordada por autores, como Nonaka y Takeuchi (1995), Wiig (1993), Zack (1999), y Bernal (2011), entre otros. Existen modelos teóricos 
y empíricos que permiten evaluar la gestión del conocimiento, y de esta manera entender y explicar la función de este concepto desde diversos puntos de vista y en diferentes contextos específicos. En la tabla 1 se presenta la propuesta de modelos de algunos autores.

Tabla 1. Modelos de gestión del conocimiento

\begin{tabular}{|c|c|c|}
\hline Autor(es) & Modelo & Descripción \\
\hline $\begin{array}{l}\text { Hedlund y } \\
\text { Nonaka } \\
(1993)\end{array}$ & $\begin{array}{l}\text { Dimensión ontológica del } \\
\text { conocimiento individual, } \\
\text { grupal, organizativo e } \\
\text { interorganizativo. }\end{array}$ & $\begin{array}{l}\text { Incluye las etapas de: almacenamiento, transferencia y transformación } \\
\text { del conocimiento. }\end{array}$ \\
\hline $\begin{array}{l}\text { Nonaka y } \\
\text { Takeuchi } \\
\text { (1995) }\end{array}$ & $\begin{array}{l}\text { Modelo del Espiral del } \\
\text { conocimiento }\end{array}$ & $\begin{array}{l}\text { Mecanismo dinámico y constante de relación entre el conocimiento tácito } \\
\text { y el explícito. Proceso de conversión del conocimiento a través de las } \\
\text { etapas de: socialización, externalización, combinación e internalización. }\end{array}$ \\
\hline $\begin{array}{l}\text { KPMG } \\
\text { Consulting } \\
(1998)\end{array}$ & $\begin{array}{l}\text { Modelo de incremento de } \\
\text { la capacidad de } \\
\text { aprendizaje en las } \\
\text { organizaciones. }\end{array}$ & $\begin{array}{l}\text { Compromiso del equipo directivo con una visión de organización } \\
\text { emprendedora, que desarrolle aprendizaje en: personas, equipo, } \\
\text { organización. Implantación de mecanismo para: creación, captación, } \\
\text { almacenamiento, transmisión y utilización del conocimiento. }\end{array}$ \\
\hline $\begin{array}{l}\text { Arthur } \\
\text { Andersen } \\
(1998)\end{array}$ & $\begin{array}{l}\text { Modelo para creación de } \\
\text { una cultura organizativa } \\
\text { orientada al aprendizaje e } \\
\text { innovación. }\end{array}$ & $\begin{array}{l}\text { Aceleración de flujo de información entre individuo y organización. } \\
\text { Novedad: perspectiva individual y organizacional. }\end{array}$ \\
\hline $\begin{array}{l}\text { Bernal } \\
(2011)\end{array}$ & $\begin{array}{lr}\text { Modelo integral de } \\
\text { administración } \\
\text { conocimiento }\end{array}$ & $\begin{array}{l}\text { Considera aspectos generales y particulares de las organizaciones; en } \\
\text { donde la gerencia o administración del conocimiento es entendida como } \\
\text { el proceso sistemático para potenciar la capacidad competitiva. } \\
\text { Dimensiones: identificación y adquisición de Conocimiento, registro y/o } \\
\text { preservación, socialización o compartición, creación y/o adaptación y } \\
\text { utilización y beneficios del conocimiento. }\end{array}$ \\
\hline
\end{tabular}

Es importante señalar que aunque la teoría de la gestión del conocimiento originada a partir del enfoque basado en los recursos y capacidades liderado por Barney (2003) y Nonaka y Takeuchi (1995) enfatizó en la empresa como objeto del análisis al explicitar que la ventaja competitiva de cada organización o empresa se encuentra en su interior, no fuera de ella, mediante el desarrollo y aprovechamiento de sus recursos y capacidades, sus actitudes, sus habilidades y, en general, sus activos intangibles y por ello los diferentes modelos iniciales de gestión de gestión del conocimiento fueron diseñados con este enfoque de empresa; enfoque que igualmente fue enriquecido con modelos particulares al mostrarse que cada contexto empresarial específico requiere de una forma diferente de gestionar el conocimiento, así como distintos sistemas de soporte a dicha gestión (Capó-Vicedo et al., 2007 y López, et al., 2015).

De otra parte, complementario a la identificación de las diferencias en los contextos empresariales como variable crítica para la gestión del conocimiento como estrategia para construir ventaja competitiva, en el actual orden económico mundial, también se evidenció la importancia de las alianzas y las aglomeraciones así como las características territoriales y económicas locales como factores claves para competir (Belussi y Sedita, 2009; Parrilli, 2009). Por este motivo, igualmente se ha identificado la necesidad de modelos de gestión del conocimiento específicos para contextos que van más allá de las fronteras de las organizaciones individuales y es así como en la actualidad se habla de modelos de gestión del conocimiento para contextos interorganizacionales como cadenas de suministro, redes empresariales, parques industriales, clusters y cadenas productivas, entre otras aglomeraciones (Cricelli y Grimaldi, 2010; Schiffauerova y Beaudry, 2012, Calamel et al., 2012).

Para Calamel et al. (2012) son diversos los ejemplos en los que se nuestra una mayor integración y difusión del conocimiento en colaboraciones entre distintas empresas de un sector de actividad que dentro de una empresa individual debido a que entre las distintas empresas se suelen crear relaciones informales, basadas en intereses comunes y en una voluntad de compartir experiencias, lo cual resulta ser mucho más efectivo a la hora de integrar y transferir conocimiento, que los procesos más formales de la empresa. Mientras que para Cricelli \& Grimaldi (2010) el trabajo colaborativo entre organizaciones del mismo sector permite a la empresas ser más innovadoras mediante la obtención y la utilización conjunta y efectiva del conocimiento.

De otra parte, Capó et al. (2005) indican que, para garantizar una interacción positiva entre las distintas empresas de un eslabonamiento que permita generar un proceso de identificación, creación e intercambio de conocimiento, es necesaria una serie de requisitos como son la similitud entre sistemas de gestión, cultura, lenguaje, objetivos, etc., que no siempre se dan entre las empresas que se relacionan. Autores estos que además agregan que, en concreto ha de conseguirse una implicación de todos los componentes de cada cadena de valor, que permita crear un clima de colaboración y mutua confianza. 
En la Tabla 2 se relacionan las metodologías propuestas para analizar la gestión del conocimiento en entornos interorganizacionales.

Tabla 2. Metodología para el análisis de la gestión del conocimiento en entornos inteorganizacionales

\begin{tabular}{|c|c|c|c|}
\hline Autor & Trabajo de Investigación & Objetivo & Metodología \\
\hline $\begin{array}{l}\text { López et } \\
\text { al. (2014) }\end{array}$ & $\begin{array}{l}\text { Procesos y prácticas de } \\
\text { gestión del conocimiento } \\
\text { en cadenas productivas de } \\
\text { Colombia. }\end{array}$ & $\begin{array}{l}\text { Determinar la capacidad de } \\
\text { las empresas colombianas } \\
\text { para gestionar su } \\
\text { conocimiento en algunas de } \\
\text { las más importantes cadenas } \\
\text { productivas del país. }\end{array}$ & $\begin{array}{l}\text { Evaluación de las competencias, los } \\
\text { procesos y las prácticas para gestionar el } \\
\text { conocimiento en empresas colombianas } \\
\text { de la cadena productiva metalúrgica, Tic, } \\
\text { de salud y agroindustria, entre otras. }\end{array}$ \\
\hline $\begin{array}{l}\text { Martínez } \\
\text { (2011) }\end{array}$ & $\begin{array}{l}\text { Desarrollo de un modelo } \\
\text { de gestión del } \\
\text { conocimiento en la cadena } \\
\text { de suministro de la } \\
\text { industria agroalimentaria. }\end{array}$ & $\begin{array}{l}\text { Diseño y validación de un } \\
\text { modelo de gestión del } \\
\text { conocimiento en la cadena de } \\
\text { suministro de la industria de la } \\
\text { harina de maíz precocida de } \\
\text { Venezuela. }\end{array}$ & $\begin{array}{l}\text { Valoración de la relación entre los } \\
\text { Índices propuestos de gestión del } \\
\text { conocimiento y de percepción de } \\
\text { resultados; teniendo en cuenta el } \\
\text { contexto de la empresa en cuanto } \\
\text { tamaño y tecnología, la gestión funcional } \\
\text { (origen, almacenamiento, transferencia, } \\
\text { aplicación y protección) del } \\
\text { conocimiento; y la gestión estratégica e } \\
\text { innovadora. }\end{array}$ \\
\hline $\begin{array}{l}\text { Solís \& } \\
\text { Pérez } \\
(2009)\end{array}$ & $\begin{array}{l}\text { Diseño de un modelo de } \\
\text { Gestión del Conocimiento } \\
\text { en el subsector de } \\
\text { plásticos para los } \\
\text { laboratorios de polímeros } \\
\text { del Centro Nacional ASTIN } \\
\text { del SENA. }\end{array}$ & $\begin{array}{l}\text { Medir el impacto del modelo } \\
\text { de gestión del conocimiento a } \\
\text { partir de las dimensiones: } \\
\text { individual, por áreas, } \\
\text { organizacional y de clientes. }\end{array}$ & $\begin{array}{l}\text { Realizar seguimiento a los indicadores } \\
\text { de Resultados No económicos } \\
\text { (Satisfacción de clientes, incremento de } \\
\text { clientes, satisfacción de los empleados, } \\
\text { calidad de productos y servicios y } \\
\text { reputación); y Resultados económicos: } \\
\text { rentabilidad crecimiento de las ventas, } \\
\text { crecimiento del beneficio, productividad } \\
\text { del trabajo, mejora en costos de } \\
\text { producción. }\end{array}$ \\
\hline $\begin{array}{l}\text { Galeano } \\
\text { et al. } \\
(2008)\end{array}$ & $\begin{array}{l}\text { Modelo de gestión del } \\
\text { conocimiento apoyado en } \\
\text { la vigilancia tecnológica y } \\
\text { la inteligencia competitiva } \\
\text { para la cadena productiva } \\
\text { de la uva isabella en la } \\
\text { bioregión del Valle del } \\
\text { Cauca. }\end{array}$ & $\begin{array}{l}\text { Proponer un modelo de } \\
\text { gestión del conocimiento en } \\
\text { las cadenas productivas del } \\
\text { Valle del Cauca que fortalezca } \\
\text { la cultura innovadora, } \\
\text { generando productos de } \\
\text { calidad y alto valor agregado } \\
\text { en la cadena de la uva } \\
\text { isabella. }\end{array}$ & $\begin{array}{l}\text { Valorar el mejoramiento de la } \\
\text { competitividad de la cadena productiva } \\
\text { de la uva isabella a partir de la } \\
\text { implementación de un sistema de } \\
\text { aseguramiento de la inocuidad, orientado } \\
\text { por el liderazgo y la gestión del } \\
\text { conocimiento como estrategia. }\end{array}$ \\
\hline
\end{tabular}

De la información de la Tabla 2 se deduce la importancia de la gestión del conocimiento en contextos y aglomeraciones o cadenas productivas específicas, situación ésta que es coherente con los planteamientos sobre cadenas productivas antes presentados.

\section{METODOLOGIA}

El diseño del modelo aquí propuesto se basa en la revisión de literatura antes descrita sobre las cadenas productivas, la gestión del conocimiento inter-organizacional y el análisis de documentos institucionales de la cadena productiva láctea del departamento de Córdoba- Colombia tales como: la Agenda prospectiva de investigación y desarrollo tecnológico en la cadena láctea colombiana (Mojica et al., 2007), el Acuerdo de competitividad de la cadena láctea colombiana (Consejo Nacional Lácteo, 2010), la Política nacional para mejorar la competitividad del sector lácteo colombiano-Documento Conpes 3675 (DNP, 2010), Consolidación de la política sanitaria y de inocuidad para las cadenas láctea y cárnica- Documento Conpes 3676 (DNP, 2010), Acuerdo regional de competitividad de la cadena productiva láctea en el departamento de córdoba y en la zona del bajo cauca antioqueño (Vega et al., 2002), entre otros y el análisis de resultados de la encuesta de gestión del conocimiento aplicada a diferentes actores de los eslabones de esa cadena.

\section{Método de muestreo}

La encuesta sobre gestión del conocimiento de escala Likert con puntuaciones de 1 a 5 (5: completamente de acuerdo, 4: de acuerdo, 3: ni de acuerdo ni en desacuerdo, 2: en desacuerdo, 1: completamente en desacuerdo) se aplicó a una muestra representada por 349 empresas de los eslabones de la cadena 
productiva de lácteos en Córdoba. Previo a su aplicación, el formulario fue sometido a consideración de un panel de académicos, expertos en el tema y actores regionales de diversos eslabones de la cadena productiva, de acuerdo a la metodología propuesta por Sánchez, Chaminade y Escobar (1999). Los datos de las encuestas fueron analizadas a través del paquete estadístico SPSS y $R$ por medio de análisis de componentes principales.

\section{Sistema de variables}

A partir de la revisión de la bibliografía sobre cadenas productivas, la información obtenida de los actores de la cadena de lácteos y del análisis de los estudios de López et al. (2014), Martínez (2011), Solís y Pérez (2009) Galeano et al. (2008), para el análisis de la gestión del conocimiento en la cadena productiva se consideraron las dimensiones y subdimensiones mostradas en las tablas 3 y 4.

Tabla 3. Dimensiones de la variable independiente gestión del conocimiento

\begin{tabular}{|c|c|c|c|}
\hline Variable & Dimensión & Subdimensión & Autores que lo proponen \\
\hline \multirow{9}{*}{$\begin{array}{l}\text { Gestión del } \\
\text { Conocimiento }\end{array}$} & \multirow{7}{*}{$\begin{array}{l}\text { Elementos } \\
\text { facilitadores de } \\
\text { la Gestión del } \\
\text { Conocimiento }\end{array}$} & Cultura Organizacional & $\begin{array}{l}\text { Sánchez et all. (2006); Velásquez (2006); Gómez } \\
\text { (2009), Tejedor y Aguirre (1998), Andersen (1998), } \\
\text { Valencia (2009) y Bernal (2011). }\end{array}$ \\
\hline & & Liderazgo & $\begin{array}{l}\text { Galeano et all. (2008); Tejedor y Aguirre (1998), } \\
\text { Andersen (1998), Euroforum (1998), Martínez (2011). }\end{array}$ \\
\hline & & $\begin{array}{l}\text { Aprendizaje } \\
\text { Organizacional }\end{array}$ & Tejedor y Aguirre (1998). \\
\hline & & Actitudes & Tejedor y Aguirre (1998). \\
\hline & & Estructura Organizativa & $\begin{array}{lllll}\text { Velásquez (2006); } & \text { Tejedor y Aguirre } & \text { (1998), } \\
\text { Euroforum (1998). }\end{array}$ \\
\hline & & $\begin{array}{l}\text { Estrategia } \\
\text { Organizacional }\end{array}$ & Velásquez (2006); Euroforum (1998). \\
\hline & & TIC & $\begin{array}{l}\text { Galeano et all. (2008); Gómez (2009), Andersen } \\
\text { (1998), Bernal (2011), Martínez (2011). }\end{array}$ \\
\hline & \multirow{2}{*}{$\begin{array}{l}\text { Gestión } \\
\text { Funcional del } \\
\text { Conocimiento }\end{array}$} & $\begin{array}{l}\text { Proceso de Creación de } \\
\text { Conocimiento }\end{array}$ & $\begin{array}{l}\text { Martínez (2011); Nonaka y Takeuchi (1995), Goñi } \\
(2000) \text {. }\end{array}$ \\
\hline & & $\begin{array}{l}\text { Almacenamiento } \\
\text { transferencia }\end{array}$ & Martínez (2011); Hedlund y Nonaka (1993). \\
\hline
\end{tabular}

Tabla 4. Dimensiones de la variable dependiente Competitividad

\begin{tabular}{|l|l|l|}
\hline Variable & Dimensión & Autores que lo proponen \\
\hline Competitividad & $\begin{array}{l}\text { Productividad (productividad y mejoras de costo de } \\
\text { producción) }\end{array}$ & $\begin{array}{l}\text { Solís y Pérez (2009); Tarí y García } \\
\text { (2011) }\end{array}$ \\
\cline { 2 - 3 } & $\begin{array}{l}\text { Rentabilidad económica (crecimiento de ventas y del } \\
\text { beneficio) }\end{array}$ & $\begin{array}{l}\text { Solís y Pérez (2009); Tarí y García } \\
\text { (2011) }\end{array}$ \\
\cline { 2 - 3 } & $\begin{array}{l}\text { Indicador Global de Éxito (Satisfacción de clientes, de } \\
\text { empleados, reputación) }\end{array}$ & $\begin{array}{l}\text { Solís y Pérez (2009); Tarí y García } \\
\text { (2011) }\end{array}$ \\
\cline { 2 - 3 } & $\begin{array}{l}\text { Inocuidad de Producto (Seguridad física, química y } \\
\text { biológica del producto) }\end{array}$ & $\begin{array}{l}\text { Galeano et all. (2008); Tarí y García } \\
\text { (2011) }\end{array}$ \\
\hline
\end{tabular}

\section{RESULTADOS Y DISCUSIÓN}

Con los datos de las encuestas, se realizó un análisis correlacional para determinar el conjunto de componentes o variables que explican la mayor variabilidad de las dimensiones de la competitividad en las empresas de la cadena. En la Tabla 5 se presentan las correlaciones entre los factores que se miden en la encuesta aplicada.

Las variables se renombraron de la siguiente forma: concepto de gestión del conocimiento (cgestcono), objetivos de la gestión del conocimiento (objgestcono), cultura organizacional (cultorg), liderazgo (lider), aprendizaje organizacional (apreorg), actitudes (actitud), estructura organizativa (estructorg), estrategia organizacional (estrategorg), tecnologías de información y comunicación (tic), proceso de creación de conocimiento (procreación), procesos de almacenamiento y transferencia de conocimiento (procalmace) y competitividad (comptt). 
Tabla 5. Matriz de correlaciones

\begin{tabular}{|c|c|c|c|c|c|c|c|c|c|c|c|c|}
\hline & 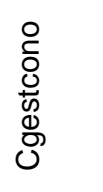 & 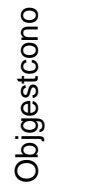 & $\begin{array}{l}\frac{D}{0} \\
\frac{0}{3} \\
0\end{array}$ & $\frac{\overline{\bar{\nu}}}{\stackrel{.}{.}}$ & $\begin{array}{l}\text { 이 } \\
\frac{0}{0} \\
\text { 인 }\end{array}$ & 总 & 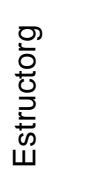 & 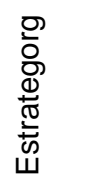 & $\stackrel{0}{i}$ & $\begin{array}{l}\text { 응 } \\
\mathbb{\Phi} \\
\frac{\mathbb{U}}{0} \\
\stackrel{0}{0}\end{array}$ & 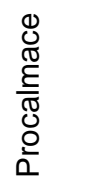 & $\begin{array}{l}\frac{F}{\overline{0}} \\
\text { हे } \\
\text { ठ }\end{array}$ \\
\hline Cgestcono & 1 & 0.544 & 0.414 & 0.534 & 0.342 & 0.370 & 0.379 & 0.380 & 0.367 & 0.537 & 0.462 & 0.148 \\
\hline Objgestcono & 0.544 & 1 & 0.392 & 0.435 & 0.356 & 0.355 & 0.187 & 0.304 & 0.357 & 0.452 & 0.552 & 0.327 \\
\hline Cultorg & 0.414 & 0.392 & 1 & 0.382 & 0.477 & 0.474 & 0.419 & 0.490 & 0.244 & 0.403 & 0.310 & 0.200 \\
\hline Lider & 0.534 & 0.435 & 0.382 & 1 & 0.307 & 0.418 & 0.401 & 0.427 & 0.335 & 0.431 & 0.416 & 0.178 \\
\hline Apreorg & 0.342 & 0.356 & 0.477 & 0.307 & 1 & 0.759 & 0.627 & 0.704 & 0.325 & 0.554 & 0.623 & 0.395 \\
\hline Actitud & 0.370 & 0.355 & 0.474 & 0.418 & 0.759 & 1 & 0.613 & 0.729 & 0.389 & 0.611 & 0.537 & 0.317 \\
\hline Estructorg & 0.379 & 0.187 & 0.419 & 0.401 & 0.627 & 0.613 & 1 & 0.700 & 0.236 & 0.594 & 0.461 & 0.352 \\
\hline Estrategorg & 0.380 & 0.304 & 0.490 & 0.427 & 0.704 & 0.729 & 0.700 & 1 & 0.420 & 0.691 & 0.546 & 0.351 \\
\hline Tic & 0.367 & 0.357 & 0.244 & 0.335 & 0.325 & 0.389 & 0.236 & 0.420 & 1 & 0.636 & 0.644 & 0.157 \\
\hline Procreacion & 0.537 & 0.452 & 0.403 & 0.431 & 0.554 & 0.611 & 0.594 & 0.691 & 0.636 & 1 & 0.740 & 0.302 \\
\hline Procalmace & 0.462 & 0.552 & 0.310 & 0.416 & 0.623 & 0.537 & 0.461 & 0.546 & 0.644 & 0.740 & 1 & 0.482 \\
\hline Comptt & 0.148 & 0.327 & 0.200 & 0.178 & 0.395 & 0.317 & 0.352 & 0.351 & 0.157 & 0.302 & 0.482 & 1 \\
\hline
\end{tabular}

El análisis de componentes principales se muestra en la Tabla 6, en donde se infiere que los primeros tres componentes (el funcional, los objetivos y los elementos facilitadores) explican el $71,6 \%$ de los resultados. Datos estos que confirman la importancia que ha de tener el componente funcional, los objetivos y los facilitadores en los procesos de la gestión del conocimiento (Martínez, 2011; Nonaka y Takeuchi, 1995; Goñi, 2000; Sánchez et al., 2006; Velásquez, 2006; Gómez, 2009; Tejedor y Aguirre, 1998; Valencia; 2009 y Bernal 2011.

Tabla 6. Componentes principales

\begin{tabular}{|c|c|c|c|}
\hline Componente & Desviación & \% de varianza & \% acumulado \\
\hline 1 & 2.273 & 0.522 & 0.522 \\
\hline 2 & 1.078 & 0.117 & 0.716 \\
\hline 3 & 0.871 & 0.076 & 0.786 \\
\hline 4 & 0.834 & 0.070 & 0.831 \\
\hline 5 & 0.663 & 0.044 & 0.901 \\
\hline 6 & 0.613 & 0.037 & 0.927 \\
\hline 7 & 0.568 & 0.032 & 0.950 \\
\hline 8 & 0.506 & 0.025 & 0.971 \\
\hline 9 & 0.470 & 0.022 & 0.988 \\
\hline 10 & 0.457 & 0.021 & 1.000 \\
\hline 12 & 0.408 & 0.016 & 0.011 \\
\hline
\end{tabular}


En la tabla 7 se presenta la asignación de componentes a las variables evaluadas; a partir de ésta asignación se renombran los factores que representan a las variables del estudio, como se indica en la tabla 8.

Tabla 7. Matriz de componentes

\begin{tabular}{|l|c|c|c|}
\hline & \multicolumn{3}{|c|}{ Componente } \\
\hline & 1 & 2 & 3 \\
\hline Cgestcono &, 635 &, 551 &,- 179 \\
\hline Objgestcono &, 667 &, 520 &,- 147 \\
\hline Cultorg &, 672 &, 029 &,- 497 \\
\hline Lider &, 696 &, 311 &,- 292 \\
\hline Apreorg &, 828 &,- 367 &,- 079 \\
\hline Actitud &, 815 &,- 245 &,- 097 \\
\hline Estructorg &, 762 &,- 448 &,- 093 \\
\hline Estrategorg &, 846 &,- 268 &,- 038 \\
\hline Tic &, 583 &, 281 &, 600 \\
\hline Procreacion &, 850 &, 015 &, 287 \\
\hline Procalmace &, 829 &, 099 &, 391 \\
\hline Comptt &, 518 &,- 231 &, 151 \\
\hline
\end{tabular}

Tabla 8. Asignación de factores

\begin{tabular}{|c|c|}
\hline Factor & Nombre \\
\hline 1 & Componente funcional \\
\hline 2 & Objetivos de la gestión del conocimiento \\
\hline 3 & Facilitadores de la gestión del conocimiento \\
\hline
\end{tabular}

\section{Propuesta de modelo}

La información de la literatura muestra que aunque existen modelos para la gestión del conocimiento en cadenas productivas específicas, las variables que en cada uno de ellos se analizan son diferentes y se complementan de tal forma que de ello se genera información suficiente y válida como para generar un modelo más integral de gestión del conocimiento aplicable a una cadena productiva como la láctea, que en el caso colombiano se caracteriza por un limitado aprovechamiento del conocimiento como recurso estratégico para la construcción de valor y acometividad sostenible.

El modelo que se propone muestra la cadena productiva de lácteos constituida por los diferentes eslabones del sector y los actores gubernamentales, académicos y agentes de la sociedad que tienen relación con esa cadena (Vila, Ferro y Rodríguez, 2000; Beckerman y Cataife, 2001 y Moncayo, 2002) y su interrelación con la gestión del conocimiento desde la perspectiva interorganizacional (Cricelli \& Grimaldi, 2010; Schiffauerova y Beaudry, 2012 y Calamel et al., 2012) representada a través de los siguientes dos componentes:

- $\quad$ Los elementos facilitadores en el cual se incluye la cultura organizacional, el liderazgo, aprendizaje organizacional, las actitudes, la estructura organizativa, estrategias y las Tic (Nonaka y Takeuchi, 1995; Capó et al, 2005, Wiig, 2009 y Bernal, 2011).

- El componente funcional, en donde se consideran de forma integral las actividades de creación, almacenamiento y transferencia del conocimiento (Bernal, 2011 y Tarí y García (2013); con el fin de avaluar la incidencia de todos estos elementos en la competitividad de la cadena y cada uno de sus eslabones, a través de la medición de la percepción de la productividad, rentabilidad, indicador de éxito e inocuidad. 
A través del modelo propuesto se busca que en su conjunto la cadena y cada agente de cada eslabón valore la incidencia de los elementos facilitadores y funcionales del proceso de gestión del conocimiento, esto porque como se mostró en la revisión bibliográfica del estudio, tanto la formalización de la cadena como la gestión del conocimiento por si solos son factores generadores de ventaja competitiva, pero mucho más cuando estos dos aspectos se conjugan y actúan de forma integrada (cadena productiva con gestión del conocimiento).

Entre las limitaciones que podrían encontrarse en la aplicación del modelo, se tiene la existencia de contadas propuestas de medición del conocimiento en entornos interorganizacionales con las cuales comparar el modelo propuesto; y el hecho de que en el modelo que se propone la medición de las variables se realiza exclusivamente mediante la recolección de datos a través de encuestas, y no a través de indicadores de capital intelectual como el cuadro de mando integral.

En la figura 2 se muestra el modelo de gestión del conocimiento propuesto para la cadena productiva de lácteos en Colombia. El modelo muestra que el concepto de cadena productiva es algo más que un eslabonamiento entre los actores de un sector productivo y que involucra actores del estado y del medio académico entre otros; y que para una adecuada gestión del conocimiento en estas cadenas productivas, es necesario tener claridad sobre los facilitadores y la funcionalidad del proceso propio de la gestión del conocimiento en contextos inter-organizacionales como estrategia específica para construir o mejorar la competitividad de la cadena.

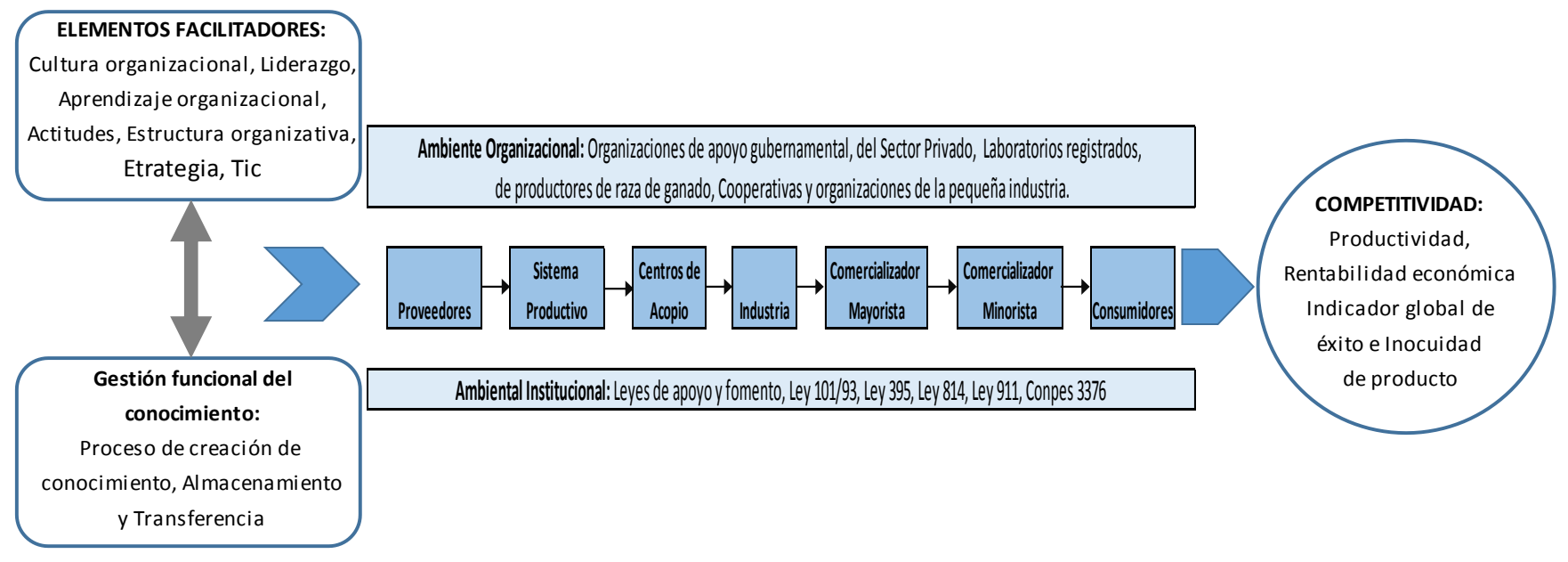

Fig. 2. Modelo de gestión del conocimiento

Las relaciones sistémicas de cooperación entre los actores de los eslabones de una cadena de producción y otros agentes o actores de la sociedad y no la acción individual de cada empresa es el factor clave para la construcción de ventajas competitivas en los sectores productivos. Las nuevas formas de cooperación (cadenas productivas) en la era de la globalización son una estrategia compleja pero efectiva, que trascienden las fronteras de las empresas y el propio sector empresarial para responder de forma competitiva a las exigencias del actual orden económico. Así, las tendencias de la flexibilización en los sistemas de producción requieren del eslabonamiento de los actores de los sectores productivos los cuales requieren de la articulación de sus actividades y la articulación de sus actividades con entidades del estado, la academia y otros actores sociales para garantizar su sostenibilidad tanto como actor y como sistema productivo.

\section{CONCLUSIONES}

La cadena productiva de lácteos en Colombia consiste en un sistema complejo de seis eslabones, donde el producto básico es la producción de leche, proveniente del ganado bovino de sistemas especializados o de doble propósito.

Para el análisis de la gestión del conocimiento en cadenas productivas se planteó un modelo teórico que define la gestión del conocimiento como independiente y la competitividad como dependiente. En la gestión de conocimiento se consideraron elementos facilitadores como: la cultura organizacional, el liderazgo, el aprendizaje organizacional, actitudes, la estructura organizativa, la estrategia organizacional y las Tic; y elementos funcionales como: el proceso de creación de conocimiento, y el almacenamiento y transferencia. Y para la variable competitividad se consideró la productividad, la rentabilidad económica, el indicador global de éxito y la inocuidad. 
La gestión del conocimiento en la cadena productiva de lácteos en Córdoba es explicada en un 71,6\% por tres factores representados por el componente funcional, los objetivos y los elementos facilitadores.

Entre las ventajas del modelo propuesto se tiene la medición de la incidencia de elementos facilitadores y funcionales del proceso de gestión del conocimiento, sobre la competitividad de la cadena y cada uno de sus eslabones; y entre las limitaciones se encuentran la medición de variables sólo mediante la recolección de datos a través de encuestas, y no a través de indicadores de capital intelectual como el cuadro de mando integral; y la existencia de contadas propuestas de medición del conocimiento en entornos interorganizacionales con las cuales comparar el modelo propuesto.

\section{REFERENCIAS}

Alavi, M. y D. E. Leidner, Review: Knowledge Management and Knowledge Management Systems: Conceptual Foundatios and Research Issues, MIS Quarterly: 25(1), 107-136 (2001).

Amit, R. y C. Zott, Value creation in e-business, Strategic Management Journal: 22(5/6), 493-520 (2001).

Andersen, A., La gestión del conocimiento en el sector sanitario. Reflexiones y retos para avanzar, Ediciones PMP (Professional Management Publications), Bilbao (1998).

Andreou, A. N., A. Green y M. Stankosky, A framework of intangible valuation areas and antecedents, doi: 10.1108/14691930710715060, Journal of Intellectual Capital (en línea), 8(1), 52-75 (2007).

Barney, J. B., Resources, capabilities, core competencies invisible assets and knowledge assets: label proliferation and theory development in the field of strategic management, 422-426, In C. E. Helfat Ed., The SIMS Blackwell Handbook of Organizational Capabilities, Oxford: Blackwell (2003).

Belussi, F. y S.R. Sedita, Life cycle vs. multiple path dependency in industrial districts, European Planning Studies: 7(4), 505-528 (2009).

Becattini, G., Del distrito industrial marshalliano a la "teoría del distrito" contemporánea: una breve reconstrucción crítica, Investigaciones regionales: 1, 19-32 (2002).

Beckerman, M. y G. Cataife, Encadenamientos productivos: Estilización e impactos sobre el desarrollo de los países periféricos, Facultad de Ciencias Económicas, Universidad de Buenos Aires, (2001), (en: [www.aaep.org.ar/espa/anales/resumen_01/bekerman_cataife.htm] -acceso: 20 de noviembre de 2015.

Benavides, C. y C. Quintana, Proceso y sistemas organizativos para la gestión del conocimiento: El papel de la calidad total, Boletín Económico de ICE No 2838, 37-52 (2005).

Bernal, C. A, Modelo de gerencia del conocimiento: visión integral, Cuadernos de Administración: 27(46), 55-66, (2011).

Briceño, M. Á. y C. A. Bernal, Estudios de caso sobre la gestión del conocimiento en cuatro organizaciones colombianas líderes en penetración de mercado, doi: 10.1016/S0123-5923(10)70140-6, Estudios Gerenciales (en línea), 26(117), 173-193 (2010).

Bueno, E., La Gestión del Conocimiento: Nuevos perfiles profesionales, Boletín del Club Intelectual 18, (en línea http://www.sedic.es/bueno.pdf, acceso: 4 de agosto 2015), (1999).

Cagliano, R., F.Caniato, y G. Spina, The linkage between supply chain integration y manufacturing improvement programmes, International Journal of Operations and Production Management: 26(3), 282-299 (2006).

Calamel, L., C. Defélix, T. Picq, y D. Retour, Inter-organisational projects in French innovation clusters: The construction of collaboration, International Journal of Project Management: 30(1), 48-59 (2012).

Calderón, J. L y F. C. Lario, Simulación de Cadenas de Suministro: Nuevas Aplicaciones y Áreas de Desarrollo, Información tecnológica: 18(1), 137-146 (2007).

Cantner, U., K. Joel y T. Schmidt, The use of knowledge management by German innovators, doi: 10.1108/13673270910971923, Journal of Knowledge Management (en línea), 13(4), 187-203 (2009).

Capó, J., M. Expósito y E. Masiá, La Gestión del Conocimiento en las redes de PYMES. El caso del cluster textil valenciano, Revista de Economía industrial: 355, 305-315 (2005). 
Capó, J., J. V. Tomás y M. Expósito, La gestión del conocimiento en la cadena de suministro: Análisis de la influencia del contexto organizativo, Información Tecnológica: 18(1), 127-135 (2007).

Capó-Vicedo, J., M.T. Martínez-Fernández, T. Vallet-Bellmunt y M. Expósito-Langa, M. Análisis de contenido de las publicaciones sobre clusters y distritos industriales en las revistas españolas, Investigaciones Europeas de Dirección y Economía de la Empresa: 17(2), 119 - 141 (2011).

Carrillo, F. J., La Evolución de las Especies de Gestión del Conocimiento: Un reporte expedicionario de los nuevos territorios, Entorno empresarial del Siglo XXI-Cinco años del Clúster del Conocimiento (en línea: http://www.knowledgesystems.org/Produccion_intelectual/reportes_tecnicos/EvolucionGC.pdf, acceso: 22 de julio 2015), España, (2001).

Castellanos, O. F., L. M. Torres y K. P. Domínguez, Manual metodológico para la definición de agendas de investigación y desarrollo tecnológico en cadenas productivas agroindustriales, 199, Giro Editores Ldta., Bogotá D.C., (2009).

Congreso de la República de Colombia, Ley 811, Bogotá D.C. (2003).

Consejo Nacional Lácteo, Acuerdo de competitividad de la cadena láctea colombiana, Serie Competitividad, (en línea: http://www.cnl.org.co/index.php?option=com_remository\&ltemid=108\&func=startdown\&id=643, acceso: 15 de junio 2015), Bogotá D.C., (2010).

Cricelli, L., y Grimaldi, M., Knowledge based inter-organizational collaborations, Journal of Knowledge Management: 14(3), 348-358 (2010).

Del Moral, A., Pazos, J., Rodríguez, E., Rodríguez-Patón, A. y Suárez, S., Gestión del Conocimiento, Paraninfo Cengage Learning, Madrid (2007).

Davenpork, T. H. y Prusak, L. Working Knowledge: How organizations manage what they know, Harvard Business School Press, Boston (1998).

Drucker, P., La sociedad post-capitalista, del capitalismo a la sociedad de conocimiento, Editorial Norma, Bogotá (1994).

Esser, K., W. Hillebrand, D. Messner y J. Jörg Meyer-Stamer, Competitividad Sistémica: nuevo desafío para las empresas y la política, Revista de la Cepal n.ำ59, Cepal, Santiago de Chile (1996).

Espinal, C. F., Covaleda, H. M. y Rodríguez, F. G., La Cadena de Lácteos en Colombia. Una mirada global de su estructura y dinámica 1991-2005, (en línea:

http://www.agronet.gov.co/www/docs_agronet/2005112162250_caracterizacion_lacteos.pdf, acceso: 20 de febrero 2015) (2005).

Euroforum, Modelo Intelect,

https://web.archive.org/web/20120428202643/http://www.gestiondelconocimiento.com/modelos.htm, acceso: 21 de agosto 2015) (2001).

Frohlich, M.T. y R. Westbrook, Arcs of integration: an international study of supply chain strategies, Journal of Operations Management: 19(2), 185-200 (2001).

Fugate, B. S., T. P. Stank y J. T. Mentzer, Linking improved knowledge management to operational and organizational performance, Journal of Operations Management: 27(3), 247-264 (2009).

Galeano, S., M. Sánchez y M. Villarreal, Modelo de gestión del conocimiento apoyado en la vigilancia tecnológica y la inteligencia competitiva para la cadena productiva de la uva isabella en la bioregión del Valle del Cauca, Cuadernos de Administración: 40, 73-93 (2008).

Gómez, M., Desarrollo de un modelo de evaluación de la Gestión del Conocimiento en empresas de manufactura, Tesis Doctoral, Universidad Politécnica de Madrid, Escuela Técnica Superior de Ingenieros Industriales (2009).

Goñi Camejo, I., Algunas reflexiones sobre el concepto de información y sus implicaciones para el desarrollo de las ciencias de la información, Acimed,8, 201-207, (2000). 
Hedlund, G. y I. Nonaka, Models of Knowledge management in the West and Japan, En Lorange, P., Chakravarthy, B., Ross, J. y Van de ven, A. (eds.): Implementing Strategic Procesess: Change, Learning and Coóperation. Basil Blackwell, Cambridge (1993).

Isaza, J., Cadenas productivas. Enfoques y precisiones conceptuales, Investigación. 8-25. En: file://C:/Users/cesarbt/Downloads/Dialnet-CadenasProductivasEnfoquesYPrecisionesConceptuales-

5137653\%20(3).pdf, acceso: 18 diciembre de 2015.

Lambert, D., M. Cooper y J. Pugh, Supply Chain Management, International Journal of Logistics Management: 9(2). 1-19 (1998).

Lima, S. M., Castro, A. M., Mengo, O., Medina, M., Maestrey, M., Trujillo, V. y Alfaro, O., La dimensión de entorno en la construcción de la sostenibilidad institucional (2001).

López, C., Redes Empresariales: Experiencias en la Región Andina, Perú: Editorial Minka, Cooperación Italiana y CEPAL (2003).

López, M., A. Hernández y C. Marulanda, Procesos y prácticas de gestión del conocimiento en cadenas productivas de Colombia, Información Tecnológica: 25(3), 125-134 (2014).

López, D., C.E. Marulanda y M. López, Métricas de Valoración de la Gestión del Conocimiento para las Pequeñas y Medianas Empresas del Sector Tecnologías de Información en el Triángulo del Café en Colombia. Información tecnológica: 26(3), 173-183 (2015).

López, C. y Meroño, Á., Strategic knowledge management, innovation and performance, doi:10.1016/j.ijinfomgt.2011.02.003, International Journal of Information Management (en línea), 31(6), 502509. (2011).

Lundvall, B., National systems of innovation. Towards a theory of innovation and interactive learning. Pinter, Londres (1992).

Martínez, M. E., Desarrollo de un modelo de gestión del conocimiento en la cadena de suministro de la industria agroalimentaria, Tesis Doctoral, Universidad Politécnica de Madrid, Escuela Técnica Superior de Ingenieros Industriales (2011).

Mojica, F. J., Cabezas, R. T., Castellanos, D. L. y Bernal, N., Agenda prospectiva de investigación y desarrollo tecnológico de la cadena láctea colombiana, 168, Ministerio de Agricultura y Desarrollo Rural, (en línea: http://www.agronet.gov.co/www/docs_agronet/200831311504_L?cteos.pdf, acceso: 15 de septiembre 2015), (2007).

Moncayo, E., Nuevos enfoques de política regional en América Latina: El caso de Colombia en Perspectiva Histórica-Las políticas regionales: un enfoque por generaciones (separata 2 de 7), Archivos de Economía N.․ 195, Departamento Nacional de Planeación, Bogotá, (2002).

Nonaka, I., y Takeuchi, H., The knowledge creating company, $1^{\text {a }}$ edición, Oxford University Press, New York (1995).

Ocampo, W. A. y Castillo, J. P., Gestión del conocimiento y Universidad como institución generadora de conocimiento, Ingeniería, (en línea

http://revistas.udistrital.edu.co/ojs/index.php/reving/article/view/2854/4150, acceso: 14 de agosto 2015), 8(1), 7 (2003).

OECD, Measuring Knowledge Management in the Business Sector: First Steps, 216, París: OECD Publishing. doi:10.1787/9789264100282 (2003).

Parrilli, M.D., Collective efficiency, policy inducement and social embeddedness: Drivers for the development of industrial districts, Entrepreneurship \& Regional Development: 21(1), 1-24 (2009).

Phan, P., D. Siegel y M. Wright, Science Parks and Incubators: Observations, Synthesis and Future Research. Journal of Business Venturing: 20, 165-182 (2005).

Porter, M., La Ventaja Competitiva de la Naciones, Editorial Plaza \& Janes, Bogotá (1991).

Porter, M., Ser competitivo, Ediciones Deusto, Barcelona, España (2009). 
República de Colombia-Departamento Nacional de Planeación, Documentos Conpes 3675: Política Nacional para mejorar la competitividad del sector Lácteo Colombiano, (en línea http://wsp.presidencia.gov.co/sncei/politica/Documents/Conpes-3675-19jul2010.pdf, acceso: 4 de agosto 2015), (2010).

República de Colombia-Departamento Nacional de Planeación, Documentos Conpes 3676: Consolidación de la política sanitaria y de inocuidad para las cadenas láctea y cárnica, (en línea http://wsp.presidencia.gov.co/sncei/politica/Documents/Conpes-3676-19jul2010.pdf, acceso: 4 de agosto 2015), (2010).

Reyes, A., Cadenas productivas: Su valor estratégico, DesignMecanico3D, (en línea http://mx.groups.yahoo.com/group/ DesignMecanico3D/message/3146, acceso: 9 de septiembre de 2015), (2005).

Salazar, F., J. Cavazos y J. Martínez, Metodología basada en el Modelo de Referencia para Cadenas de Suministro para Analizar el Proceso de producción de Biodiesel a partir de Higuerilla, Información tecnológica: 23(1), 47-56 (2012).

Sánchez, M., Chaminade, C. y Escobar, C., En busca de una teoría sobre la medición y gestión de los intangibles en la empresa: una aproximación metodológica, Ekonomiaz: Revista vasca de economía: (45), 188-213 (1999).

Sánchez, S., M. Trillo, C. Mora y M. Ayuso, La cultura organizacional como núcleo central en la medición del capital intelectual, Revista de Psicología del Trabajo y de las organizaciones: 22 (2), 179-202, (2006).

Schiffauerova, A. y C. Beaudry, Collaboration spaces in Canadian biotechnology: A search for gatekeepers. Journal of Engineering and Technology Management: 29, 281- 306 (2012).

Simanca, M. y Montoya, L., Análisis del agronegocio de la cadena productiva de lácteos, Rev. Fac. Nac, Agr: 67(2), 1071-1074 (2014).

Solís, M. y Pérez, B., Modelos de Gestión del Conocimiento. El caso de los laboratorios del Centro Nacional ASTIN del Sena, Revista Informador Técnico: 73, 44-52 (2009).

Soto, M., y Barrios, N., Gestión del conocimiento. Parte I. Revisión crítica del estado, Acimed, 14(2), 43, (2006).

Storper, M. Sociedad, comunidad y desarrollo económico. Ekonomiaz Revista vasca de economía: 58, 1243 (2005).

Tarí, J. J. y M. García, ¿Puede la gestión del conocimiento influir en los resultados empresariales?, doi: 10.5295/cdg.I00263jt, Cuadernos de Gestión (en línea), 13(1), 151-176. (2011).

Tejedor, B. y A. Aguirre, Proyecto Logos: Investigación relativa a la capacidad de aprender de las empresas Españolas, Boletín de Estudios Económicas: 53 (164), 231-249 (1998).

Valencia, M., Modelo para la creación del conocimiento para Pymes, Entramado: 5(2), 10-27 (2009).

Vega, A., A. Martínez y L. Meneses, Acuerdo regional de competitividad de la cadena productiva láctea en el departamento de Córdoba y en la zona del Bajo Cauca Antioqueño, 43, Centro de Investigaciones Turipaná Corpoica Regional, Cerete-Córdoba (2012).

Velásquez, L., Gestión del conocimiento en el contexto de organizaciones que aprenden (En línea http://www.cidar.uneg.edu.ve/DB/bcuneg/EDOCS/TESIS/TRABAJOS_DE_ASCENSOS/TASHDV45L852006 Luis\%20Velasquez.pdf, acceso: 18 de septiembre de 2015), Universidad Nacional Experimental de Guayana, República Bolivariana de Venezuela (2006).

Vila, M., C. Ferro y M. Rodríguez, Agrupamientos sectoriales territoriales (A.S.T.): reflexiones acerca de los recursos compartidos, Revista de Economía y Empresa: XIV (40), 87-101 (2000).

Wiig, K. M., Knowledge Management Foundations: thinking about-how people and organizations create, represent, and use knowledge, Arlington, Texas: Schema (1993).

Zack, M. H., Managing codified knowledge, Sloan management review: 4O(4), 45-58 (1999). 